\title{
Article \\ Effect of Water and Salt on the Colloidal Stability of Latex Particles in Ionic Liquid Solutions
}

\author{
Dóra Takács ${ }^{1} \mathbb{1}$, Matija Tomšič ${ }^{2}\left(\mathbb{D}\right.$ and Istvan Szilagyi ${ }^{1, *} \mathbb{( D}$ \\ 1 MTA-SZTE Lendület Biocolloids Research Group, Department of Physical Chemistry and Materials Science, \\ University of Szeged, Rerrich Béla tér 1, H-6720 Szeged, Hungary; takacs.dora@szte.hu \\ 2 Faculty of Chemistry and Chemical Technology, University of Ljubljana, Večna pot 113, \\ 1000 Ljubljana, Slovenia; Matija.Tomsic@fkkt.uni-lj.si \\ * Correspondence: szistvan@chem.u-szeged.hu
}

check for updates

Citation: Takács, D.; Tomšič, M.; Szilagyi, I. Effect of Water and Salt on the Colloidal Stability of Latex Particles in Ionic Liquid Solutions. Colloids Interfaces 2022, 6, 2. https:// doi.org/10.3390/colloids6010002

Academic Editor: habil. Reinhard Miller

Received: 26 November 2021 Accepted: 29 December 2021 Published: 31 December 2021

Publisher's Note: MDPI stays neutral with regard to jurisdictional claims in published maps and institutional affiliations.

Copyright: (c) 2021 by the authors. Licensee MDPI, Basel, Switzerland. This article is an open access article distributed under the terms and conditions of the Creative Commons Attribution (CC BY) license (https:/ / creativecommons.org/licenses/by/ $4.0 /)$.

\begin{abstract}
The colloidal stability of sulfate (SL) and polyimidazolium-modified sulfate (SL-IP-2) latex particles was studied in an ionic liquid (IL) of ethylammonium nitrate (EAN) and its water mixtures. Aggregation rates were found to vary systematically as a function of the IL-to-water ratio. Repulsive electrostatic interactions between particles dominated at low IL concentrations, while they were significantly screened at intermediate IL concentrations, leading to destabilization of the dispersions. When the IL concentration was further increased, the aggregation of latex particles slowed down due to the increased viscosity and finally, a striking stabilization was observed in the IL-rich regime close to the pure IL solvent. The latter stabilization is due to the formation of IL layers at the interface between particles and IL, which induce repulsive oscillatory forces. The presence of the added salt in the system affected differently the structure of the interfaces around SL and SL-IP-2 particles. The sign of the charge and the composition of the particle surfaces were found to be the most important parameters affecting the colloidal stability. The nature of the counterions also plays an important role in the interfacial properties due to their influence on the structure of the IL surface layers. No evidence was observed for the presence of long-range electrostatic interactions between the particles in pure ILs. The results indicate that the presence of even low concentrations of water and salt in the system (as undesirable impurities) can strongly alter the interfacial structure and thus, the aggregation mechanism in particle IL dispersions.
\end{abstract}

Keywords: ionic liquid; aggregation; latex particle; light scattering; colloidal stability

\section{Introduction}

Room temperature ionic liquids (ILs) are a class of tunable and extraordinarily versatile solvents composed solely of ions and hence, they possess a variety of properties making them interesting for numerous applications [1,2]. For instance, they have advantageous characteristics such as negligible vapor tension, nonflammability, wide electrochemical window, high boiling, and low freezing points. Therefore, their operational range is quite imposing. In addition, they possess interesting solvation and interfacial properties. Thus, developing particle dispersions in ILs is a continuously evolving field since the physicochemical properties of the IL-(nano)particle dispersions can be superior compared to the ones of the one phase counterparts [3]. This improvement strongly depends on the balance of bulky and interfacial interactions between ILs and the particles [4,5]. Applications of IL-particle dispersed systems include producing functional nanomaterials [6,7], catalytic reactions [8-10] and various electrochemical processes [1,11], to name a few. Since the importance of these utilizations has begun to grow, the fundamental research must focus on the understanding of the mechanisms behind the stabilization of (nano)particles in ILs. While the properties of bulk ILs often define the solvent physicochemical parameters, when used as the solvent, these properties may be altered at the solid-IL interface due to the denser ion structuration near to the charged surface [12-14]. 
In general, the stability of colloidal systems is known to be governed by a balance of attractive and repulsive forces, which are well described for aqueous dispersions by the classical Derjaguin, Landau, Verwey, and Overbeek (DLVO) theory [15-17]. To prevent the particle aggregation in these colloids, the surface of the particles can be tailored to enhance the strength of the repulsive forces enough to overcome the van der Waals attraction and keep the particles separated [16]. This repulsion can be obtained either electrostatically or sterically or it can result from the combination of these two effects (electrosteric) [16]. Such stabilization can be achieved by modifying the surface of the particles with polymeric substances [15,18]. Nevertheless, ILs are fundamentally different in relation to the solvent properties of the polar liquids, since the electrostatic stabilization is typical for the polar molecular liquids is unlikely to occur in them, as their strong ionic character prevents the manifestation of the classical long-range repulsive interparticle interactions due to their strong charge screening $[19,20]$. Therefore, the proposed stabilizing mechanism in ILs is the so-called solvation force. In this situation, a solvation layer of IL ions forms around the particles resulting in repulsive interparticle interactions [6,21,22].

Experiments have recently demonstrated that the nanoscale features of ILs may be useful for controlling the stability of particle dispersions. The scattering measurements and molecular dynamic simulations revealed that many ILs are nanostructured in bulk [23-25] and the surface force measurements have demonstrated that this structuring manifests as layering, when ILs get in contact with solid surfaces [14,26,27]. Therefore, it is evident that the interfacial assembly of the IL ions around the particles has an important stabilization role in the particle suspension, since it greatly influences the interactions between the surfaces $[18,28]$. Besides, the IL constituents have distinct affinity to a given surface, so they can modify the surface charge in different extent, which affects the aggregation process in the media applied [22,29-31]. Therefore, to possibly tune the colloidal stability of an IL based dispersion, the parameters, which affect the strength on interactions between IL constituents and the solid surface, should be explored and optimized.

The properties of the solid-liquid interfaces are determined by the features of both the surface and the liquid. However, the surface composition can be further altered by introducing external influences, such as changes in ionic strength, temperature, or $\mathrm{pH}$. In addition, many applications of ILs require homogeneously dispersed (nano)particles across a broad range of conditions $[4,5,32]$. Therefore, the consequences caused by the undesirable presence of water and/or salt impurities in such systems must be clarified. It is assumed that these components can strongly alter the interfacial structure of the solid-IL interface, since the IL solvation layering is disrupted in their presence [33-35]. Despite the absence of the aforementioned long-range electrostatic interactions in ILs, the charge of the surface and of the ions present determines the composition and structure of the adsorbed interfacial layer. Unfortunately, only a limited number of studies have been performed investigating the effect of added salt on the stability of IL based colloidal systems. For example, it has been shown, that the presence of $\mathrm{Li}^{+}$in a silica-IL colloidal dispersion modifies the microstructure of the system and has a strong influence on the structuring of the interface [33]. Nevertheless, there is a lack of extensive research studies performed on IL-based colloidal dispersions containing additionally dissolved salts.

The scope of this work is to contribute to the fundamental understanding of the structuring features of solid-IL interface by focusing on its response to external influences, as the addition of water and $\mathrm{Na}^{+}$salt to the colloidal system. Both the dispersed phase, in a form of the dispersed latex particles, and the IL solvent phase, represented by the pure ethylammonium nitrate (EAN), have been individually studied before, but their mixed system provides an excellent platform to explore fundamental charging and aggregation processes in such dispersions. EAN was chosen as it possesses some properties (e.g., relatively moderate viscosity and refractive index, low melting point), which makes it suitable for light scattering based studies as the present one. While latex particles have proved to be appropriate model particles to study fundamental aggregation processes. Therefore, this paper provides an improved understanding of how the above-mentioned factors govern the 
interfacial composition, as the presented findings offer tools for tuning and optimizing the stability of IL-particle dispersions for specific applications. Note that surface charges and aggregation rates were also explored by our group in other particle-IL systems, but those studies concentrated on particles dispersed in diluted aqueous IL solutions [30,31,36,37]. The present work focuses on a much wider range of IL concentration (up to pure EAN) and on the effect of both water and salt content on the colloidal stability of the dispersions.

\section{Materials and Methods}

\subsection{Materials}

Spherical sulfate functionalized polystyrene latex particles (SL) were purchased from Thermo Fischer Scientific. The particles have a mean radius of $215 \mathrm{~nm}$, a polydispersity of $1.8 \%$ expressed as coefficient of variation and a charge density of $12 \mathrm{mC} / \mathrm{m}^{2}$. These quantities were determined by the manufacturer using transmission electron microscopy (TEM) and conductometric titration, respectively. Dynamic light scattering (DLS) yielded slightly higher hydrodynamic radii $(257 \mathrm{~nm})$ in stable suspensions, due to the finite polydispersity of the original samples, since the particles of higher size contribute much more to the scattered intensity in the DLS measurements, than the smaller ones. Note that the negative charge of the particles is compensated with $\mathrm{K}^{+}$counterions.

The polyimidazolium-based polymer (IP-2) with $\mathrm{Cl}^{-}$counterions was synthesized according to the protocol described elsewhere [38]. The immobilization of the IP-2 polymer onto the surface of SL particles (denoted as SL-IP-2 thereafter) was achieved by simple mixing of proper volumes of ultrapure water, IP-2 and SL particle samples. The stock suspensions were subsequently diluted to $20 \mathrm{mg} / \mathrm{L}$ for light scattering measurements and set to $\mathrm{pH} 4$ by $\mathrm{HCl}$ (VWR). During sample preparation, ultrapure water was obtained from Puranity TU+ (VWR) apparatus. Prior to use, the water and the electrolyte solutions were filtered with $0.1 \mu \mathrm{m}$ syringe filters (Millex) to prevent dust contamination.

Ethylammonium nitrate (EAN) was purchased from IoLiTech GmbH (Heilbronn, Germany). It belongs to the class of room-temperature ILs, and it is completely miscible with water in all concentration ratios. For analysis of DLS measurements, the viscosity and refractive index data for EAN-water mixtures were taken from the literature [39]. The concentration of pure EAN is $11.6 \mathrm{~mol} / \mathrm{L}$, while the water content was below $3 \mathrm{~g} / \mathrm{L}$ (which corresponds to $0.17 \mathrm{M}$ ), as determined by Karl-Fischer coulometric titrations using a KEM MKC-710 Karl Fischer Moisture Titrator. Sodium nitrate $\left(\mathrm{NaNO}_{3}\right)$ used as background electrolyte was purchased from AnalR NORMAPUR.

\subsection{Dynamic Light Scattering}

Time-resolved DLS was applied to follow the possible aggregation processes in the samples. The measurements were performed using a NIBS High-Performance Particle Sizer (ALV GmbH) equipped with a $3 \mathrm{~mW}$ He-Ne laser of $633 \mathrm{~nm}$ wavelength and the scattering angle was $173^{\circ}$. The samples were prepared by mixing the appropriate amount of water with EAN and salt solutions to achieve the desired IL and $\mathrm{NaNO}_{3}$ concentrations. The experiments were initiated by adding the aqueous particle suspension to the IL-water mixture in the cuvette, followed by rapid mixing. The final particle concentration was $20 \mathrm{mg} / \mathrm{L}$, which was sufficiently low that the particle aggregation endured in its early stages and the contribution of the higher order aggregates could be neglected. This method limited the lowest achievable water content to about $3 \mathrm{~g} / \mathrm{L}$.

DLS probes the fluctuations of the scattered light and evaluates the dependence of the intensity correlation function with time. Each correlation function was recorded for $20 \mathrm{~s}$ and it was fitted with cumulant method to obtain the decay rate constant $(\Gamma)$ [40]. From this constant, one can extract the translational diffusion coefficient $(D)$, which is converted to hydrodynamic radius $\left(R_{h}\right)$ by means of the Stokes-Einstein relation [41]

$$
D=\frac{\Gamma}{q^{2}}=\frac{k_{B} T}{6 \pi \eta R_{h}}
$$


where $\eta$ is the viscosity of the media, $T$ is the absolute temperature, $k_{B}$ is the Boltzmann constant, and $q$ is the magnitude of the scattering vector. The apparent dynamic rate constant $(\Delta)$, which describes the initial increase of $R_{h}$, is given as $[40,42]$

$$
\Delta=\frac{1}{R_{h}(q, 0)} \cdot\left(\frac{d R_{h}(q, t)}{d t}\right)_{t \rightarrow 0}=k N_{0}\left(1-\frac{1}{\alpha}\right) \frac{I_{2}(q)}{2 I_{1}(q)}
$$

where $t$ is the experimental measurement time, $N_{0}$ is the initial number concentration of the particles $\left(4.55 \times 10^{14} \mathrm{~L} / \mathrm{m}^{3}\right), k$ is the absolute aggregation rate coefficient (in $\mathrm{m}^{3} / \mathrm{s}$ unit), and $\alpha$ is the hydrodynamic factor. This factor represents the ratio between the diffusion coefficients of the monomer $\left(D_{1}\right)$ and the dimer $\left(D_{2}\right)$ and its numerical value can be estimated from calculation of the friction coefficient of a dimer under laminar flow conditions $\left(\alpha=D_{1} / D_{2}=1.39\right)$ [43]. The contribution of the form factors of the monomer $I_{1}(q)$ and the dimer $I_{2}(q)$ to the scattered intensity was predicted by the Rayleigh-GansDebye approximation as $I_{2}(q) / 2 I_{1}(q)=1+\left(\sin \left(2 q R_{1}\right) / 2 q R_{1}\right)$, where $R_{1}$ is the radius of the particle $[17,40]$. In the early stages of aggregation, only a dimer formation is assumed, until the $R_{h}$ does not increase more than $40 \%$ in these measurements. The value of $\Delta$ can be obtained from the initial slope of the apparent $R_{h}$ versus time, as shown in the Figure 1. Note that $R_{h}$ always increases with time in an aggregating suspension, as one would intuitively expect. The standard error of the obtained $\Delta$ values was about $8 \%$.
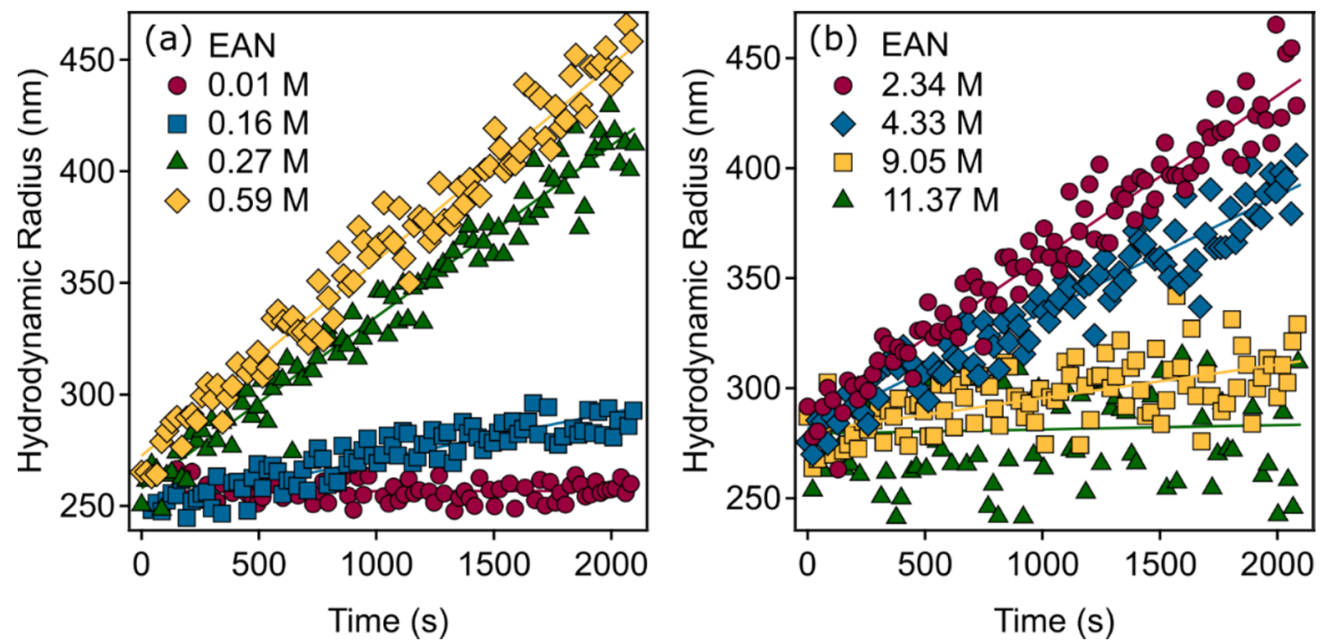

Figure 1. Time-dependence of the hydrodynamic radii measured by DLS for SL particles in EANwater mixtures at different EAN concentrations on the water-rich side (a) and on the IL-rich side (b). The solid lines present the linear fits to the data used to calculate the aggregation rate constants according to Equation (2).

\subsection{Phase Analysis Light Scattering}

Continuously monitored phase analysis light scattering (cmPALS) measurements were performed with a Litesizer 500 instrument (Anton Paar) at a scattering angle of $175^{\circ}$ to determine the electrophoretic mobility of the particles in IL-water mixtures. The applied voltage during the measurements was $200 \mathrm{~V}$. In operation, the light source is producing coherent primary light beam, which is split by a beam splitter. One part is sent to the oscillating modulator and the other part is used in the light scattering experiment. The scattered light is then combined with the modulated reference beam from the modulator and detected on the primary scattering detector. Additionally, also a smaller part of the modulated reference beam from the modulator is monitored continuously by a secondary detector and used for the corrections of possible instabilities in modulator frequency. The resulting monitor trace represents the reference beam, while the detector trace shows the interference (modulation) between the scattered light from the sample and the modulated reference beam. The obtained phase plot shows the phase difference between the detector 
and the monitor traces due to the Doppler effect on the scattered light by moving scattering particles and the fit of the cmPALS to the data, from which the electrophoretic mobility values were determined. This parameter quantifies the ratio between the travel velocity of the particle relative to the applied electric field. The sample preparation was identical as in the DLS measurements with the exception that the samples were allowed to rest for $2 \mathrm{~h}$ at room temperature before the measurements, which occurred after 1 min equilibration time in capillary cuvettes or with the Univette accessory (Anton Paar). The latter measuring cell is recommended for high conductivity samples; therefore, it was used to measure samples containing ILs. Five runs were performed and averaged.

\section{Results and Discussion}

The aggregation kinetics and charging characteristics of bare and IP-2 functionalized SL particles dispersed in IL-water mixtures in the concentration range from dilute solution of EAN in water to neat EAN containing only a small amount of water were investigated by light scattering measurements. The effect of added $\mathrm{NaNO}_{3}$ salt on the colloidal stability of these systems was also assessed. The principal aim of these experiments determining the electrophoretic mobilities and aggregation rates was to clarify the behavior of EAN at the solid-liquid interface and to study the sensitivity of the surface compositions to external influences, such as addition of water or salt.

\subsection{IP-2 Functionalization of $S L$}

Commercially available SL particles were functionalized with oppositely charged IP-2 polymer. The aim was to find the optimal experimental conditions for stable dispersion of SL-IP-2 particles coated with the polymer. The $\mathrm{pH}$ of the samples was kept at 4.0, and the dispersions contained $10 \mathrm{mM} \mathrm{KCl}$ as a background electrolyte. Electrophoretic mobility measurements were performed to follow the adsorption of the polymer on the particle surface and the resulting data recorded at various IP-2 doses are shown in Figure 2.

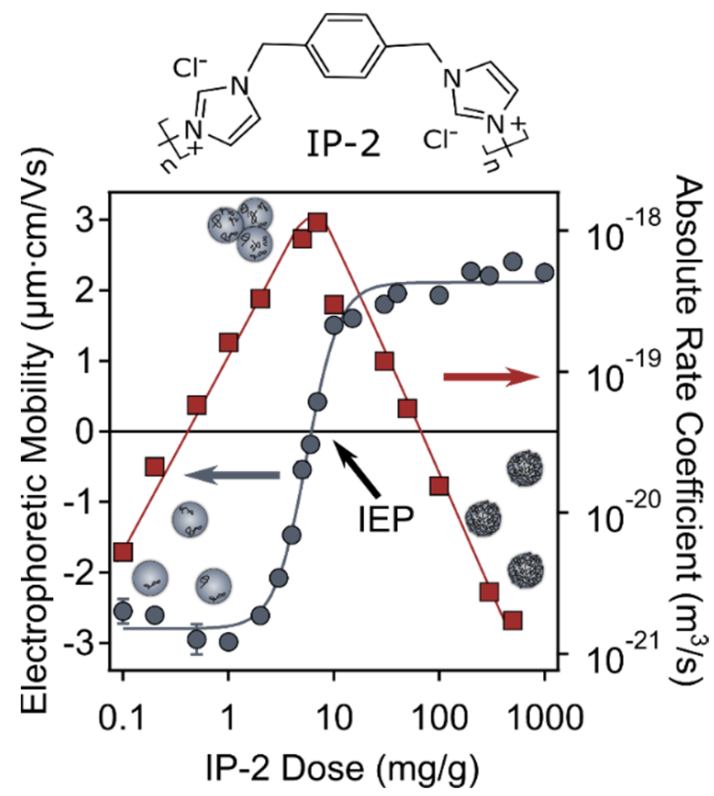

Figure 2. Electrophoretic mobility (circles, left axis) and absolute rate coefficient (squares, right axis) values of SL particles as a function of the IP-2 dose. The measurements were performed in aqueous dispersions at $\mathrm{pH} 4$ and $10 \mathrm{mM}$ ionic strength adjusted by $\mathrm{KCl}$. The unit $\mathrm{mg} / \mathrm{g}$ refers to $\mathrm{mg} \mathrm{IP}-2$ per one gram of SL. The arrows indicate the axis, to which mobility (blue arrow) and aggregation rate (red arrow) data points belong. The lines serve to guide the eye. The upper part of the figure shows the structure of IP-2 polymer. 
In general, the mobilities changed from negative to positive by increasing the added amount of IP-2 polymer, indicating its strong affinity to the oppositely charged particle surface. The mobility values were negative at low doses, since the polymer was able to only partially compensate the surface charge of the particles at these conditions. A further increase in the IP-2 dose led to the charge neutralization at the isoelectric point (IEP), where the particles have zero net charge. The adsorption process continued at higher doses and subsequent charge reversal of the particles occurred leading to positive net charge on the particles. At the highest doses applied, the mobility values reached a plateau with an onset around a $200 \mathrm{mg} / \mathrm{g}$ dose. Note that the coated particles possess significantly lower magnitude of mobility at the plateau than the bare particles indicating a lower surface charge density in the former case.

Furthermore, the absolute aggregation rate coefficients were determined by timeresolved DLS measurements under the same experimental conditions as the electrophoretic mobilities to explore particle aggregation processes in the dispersions. Figure 2 indicates that the gradual decrease in the mobility values is in line with the changes in the absolute aggregation rates. Accordingly, when the particles possess high electrophoretic mobility values (either negative or positive), the aggregation rate values are low indicating the stable dispersions or slow aggregation. However, at IP-2 doses near to the IEP, the rate values increase indicating a rapid particle aggregation and hence, unstable samples. Such a charging and aggregation behaviors resemble the one observed earlier in oppositely charged polyelectrolyte-particle systems $[31,36,44,45]$ and are in qualitative agreement with the DLVO theory, which predicts the stable dispersions in case, when the surface charge is high and the corresponding repulsive double layer forces prevail the attractive van der Waals interactions [46].

Based on these results, the particles prepared at a dose of $500 \mathrm{mg} / \mathrm{g}$ (sample denoted as SL-IP-2) were used for the further studies. At this composition, the SL particle surface is fully coated with the polymer, while the high positive charge and very low aggregation rate ensure appreciable colloidal stability of the SL-IP-2 sample. Therefore, in the following, the negatively charged particles are referred to as SL and positive ones as SL-IP-2 particles.

\subsection{Generic Trend in Aggregation Kinetics}

First, the aggregation characteristics of the SL and SL-IP-2 particles in EAN and in its water mixture systems were explored. Figure 3 indicates that in general, three main aggregation regimes were identified in both cases. Namely, at the water-rich side, i.e., up to 1.0 M EAN concentration, the aggregation rates were initially low, but they increased rapidly with increasing IL contents until they reached the plateau values. This tendency is consistent with the DLVO theory predictions and hence, it was named as DLVO regime. Accordingly, the initial increase of the aggregation rate values corresponds to the slow aggregation region, in which the trend is mainly governed by the progressive charge screening by the EAN ions and simultaneous surface adsorption of the counterions. Note that for the negatively charged SL particles ethylammonium cations, while for the positively charged SL-IP-2 nitrate anions were the counterions. The plateau at higher concentrations of EAN refers to the fast aggregation region, where the percentage of particle collisions, which lead to dimer formation only depends on the rate of their diffusion in the given media. The obtained absolute aggregation rates in this fast aggregation regime $\left(\mathrm{k}_{\text {fast }}\right)$ were found to be $2.63 \times 10^{-18} \mathrm{~m}^{3} / \mathrm{s}$ and $5.12 \times 10^{-18} \mathrm{~m}^{3} / \mathrm{s}$ for the SL and SL-IP-2 particles, respectively. 

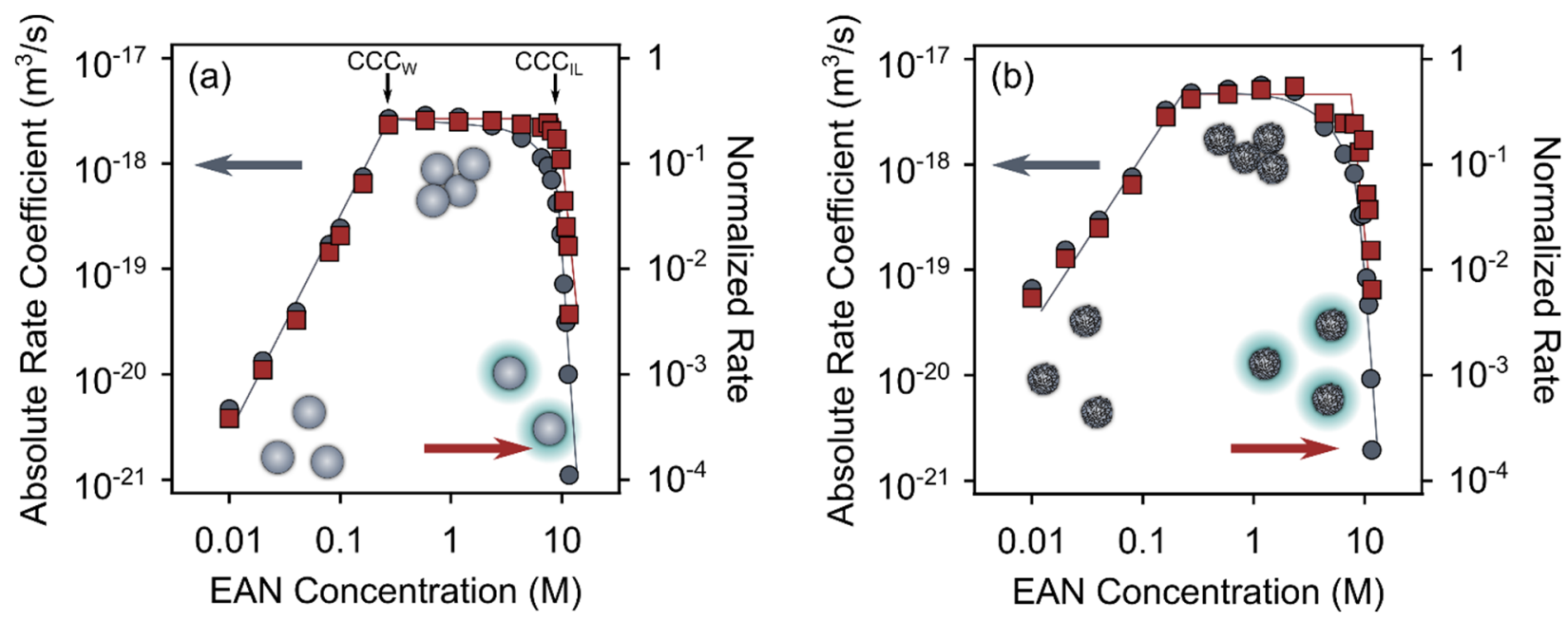

Figure 3. Absolute aggregation rate coefficients (circles, left axes) and normalized aggregation rate coefficients (squares, right axes) for SL (a) and SL-IP-2 (b) particles in water. In (a) the $C C C_{S}$ corresponding to the water-rich $\left(C C C_{W}\right)$ and EAN-rich $\left(C C C_{I L}\right)$ concentration regimes are indicated. The solid lines serve to guide the eye.

This scenario is similar to the one induced by the simple inorganic salts [30,47,48], since the ILs tend to fully dissociate and hydrate in dilute aqueous solutions, similar as observed for the inorganic electrolytes $[21,30,49]$. The sharp transition between the reported fast and slow aggregation regimes is located at the so-called critical coagulation concentration (CCC). Therefore, this parameter can quantify the destabilization power of the given solvent medium. The obtained $C C C$ values in the water-rich regime $\left(C C C_{W}\right)$ are expressed as molar concentrations of EAN (Table 1), but those in the IL-rich regime $\left(C C C_{I L}\right)$ are expressed as molar concentration of water. They were determined from the data presented in Figure 3 and are gathered in Table 1. The presented findings are generic for both SL (see Figure 3a) and SL-IP-2 particles (see Figure 3b).

Table 1. Characteristic aggregation data of the particles measured in EAN-water mixtures.

\begin{tabular}{|c|c|c|c|c|}
\hline & \multicolumn{2}{|c|}{ SL } & \multicolumn{2}{|c|}{ SL-IP-2 } \\
\hline & $\operatorname{CCC}_{W}{ }^{\mathrm{a}}$ & $C C C_{I L}{ }^{b}$ & $\operatorname{CCC}_{W}{ }^{\mathrm{a}}$ & $C C C_{I L}{ }^{b}$ \\
\hline EAN & 0.23 & 7.1 & 0.15 & 15.0 \\
\hline $\mathrm{EAN}-0.001 \mathrm{M} \mathrm{NaNO}$ & 0.16 & 8.5 & 0.15 & 12.6 \\
\hline $\mathrm{EAN}-0.01 \mathrm{M} \mathrm{NaNO}_{3}$ & 0.15 & 7.3 & 0.14 & 16.2 \\
\hline $\mathrm{EAN}-0.1 \mathrm{M} \mathrm{NaNO} 3$ & 0.05 & 7.8 & 0.05 & 14.8 \\
\hline
\end{tabular}

a Concentration of EAN in M. ${ }^{\mathrm{b}}$ Concentration of water in M. The accuracy of the CCC determination method is about $10 \%$.

In the second regime, i.e., at intermediate EAN concentrations above $1.0 \mathrm{M}$, the decrease in the aggregation rates was gradual. This trend arises due to the increasing viscosity of the mixture. Nevertheless, the aggregation remains diffusion controlled. Therefore, this regime was designated to exhibit the viscous stabilization. To consider such a stabilization mechanism the aggregation rates were normalized by the Smoluchowki rate $\left(k_{S}\right)$, which takes into account the dynamic viscosity of the solution for diffusion-controlled aggregation as follows [22]

$$
k_{S}=\frac{8 k_{B} T}{3 \eta}
$$

Since the viscosities of the mixtures strongly increase with increasing IL concentration, in parallel the Smoluchowski's value decreases. Therefore, the normalized rate coefficient $\left(k / k_{S}\right)$ remains constant in the entire viscous stabilization regime, which proves that the gradual slowdown in the aggregation is caused by the increased viscosity of the media and 
that the aggregation is diffusion controlled [48]. Note that the experimentally obtained aggregation rate coefficients $(k)$ were smaller than the calculated values $\left(k_{S}\right)$ by a factor around $2-3$ in the fast aggregation regime. This discrepancy is because absolute rate constants are influenced by hydrodynamic and van der Waals interactions, while Smoluchowski's theory neglects them. Similar phenomena were observed in aqueous dispersion of other colloidal particles $[50,51]$.

Finally, in the last regime, upon further increasing the IL concentration, one observes a rapid decrease of the aggregation rates. This phenomenon was denoted as solvation stabilization. In this regime, the samples contained only a small concentration of water. Since the stabilizing mechanism in IL media lies on the formation of solvation layers (which will be further discussed later), the water present in the samples can disrupt the layering of IL constituents at the particles surface. Similar interfering role of water at the interface was reported in other works as well $[22,52,53]$. The onset of the third characteristic regime is identified by the sudden decrease of the normalized aggregation rates at high IL concentration. Here, a similar critical coagulation concentration $\left(C C C_{I L}\right)$ can be identified as in the case of DLVO regime. Therefore, $C C C_{I L}$ represents the transition between the viscous and solvation stabilization regimes and is expressed in molar concentration of water.

The obtained $C C C_{I L}$ values (see Table 1) indicate that for the destabilization of the particles in the EAN-rich regime, the higher concentration of water is needed in the case of SL-IP-2 particles. This most probably originates from the fact that EAN is a good solvent for the coating polymer and creates a solvation sphere with polymer tails and loops around the SL particle, which manifests itself as an additional stabilizing contribution of steric origin acting between the adsorbed IP-2 layers [54].

In addition, one also observes that the magnitude of the aggregation rate coefficients in the intermediate regime for the SL-IP-2 particles is much higher than for the SL particles. This difference occurs due to the significantly higher surface charge of the bare SL particles (see Figure 2), which results in somewhat stronger electrostatic repulsion between the particles, i.e., in slower aggregation.

\subsection{Charging Characteristics}

To further investigate the behavior of ILs upon interaction with charged particles, the electrophoretic mobility values for both particles in the whole range of EAN-water mixtures were determined. It should be noted that the acidity conditions before the particle transfer to the IL-water mixtures are of great importance, because they determine the initial surface charge of the particles that is crucial from the aspect of the colloidal stability, i.e., charging feature affect the aggregation processes in terms of the nature or origin of interparticle forces [32]. Under the given experimental conditions $(\mathrm{pH} 4)$ the SL particles have negative charge, but the SL-IP-2 particles are positively charged.

Figure 4a shows that although the electrophoretic mobility values are specific to the given particles, the trends in the magnitudes are very similar in both cases. Accordingly, their absolute value initially decreases with increasing the EAN concentration due to the surface charge screening and eventually settles very close to zero at higher EAN concentrations. This behavior is typical for charged colloids suspended in monovalent salt solutions and can be well-explained with the traditional theories developed for the description of the electrical double layer [30,55]. In addition, the phase plots shown in Figure $4 \mathrm{~b}$ provide information about the motion of the particles in the given media. One can observe that in the water-rich regime the charged particles tend to move towards the electrode of opposite polarity. However, on the IL side, where solvation stabilization occurs, no particle movement could be detected by applying the electric field during the cmPALS measurements. 

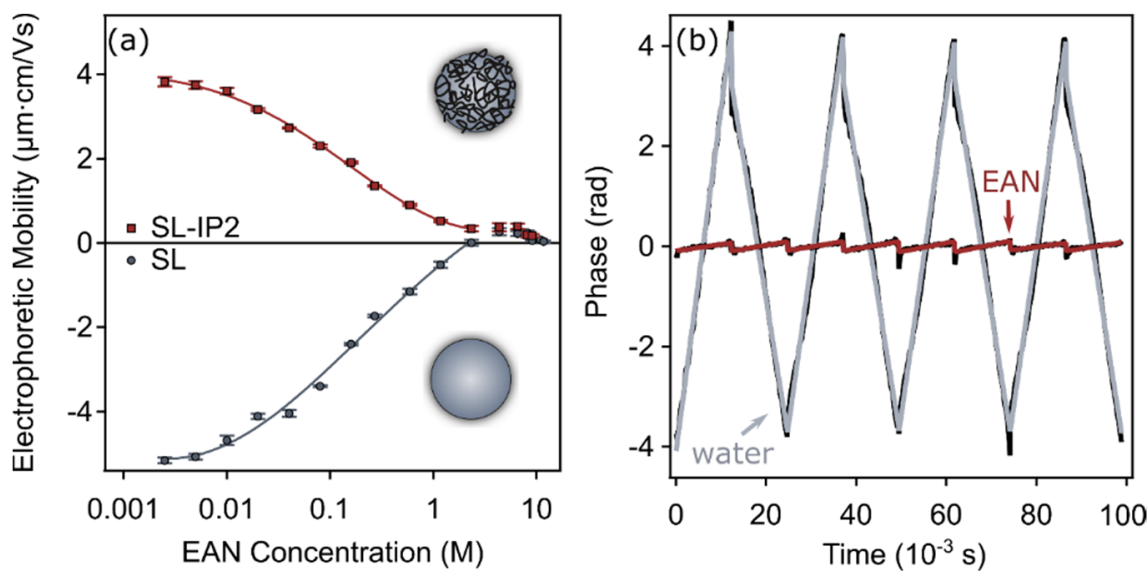

Figure 4. Charging characteristics of polystyrene latex particles in EAN-water mixtures. Electrophoretic mobility values of SL and SL-IP-2 particles at different EAN concentrations (a) and the obtained phase plots in the presence of SL particles on the water-rich side ( $2.5 \mathrm{mM}$ EAN concentration) and IL-rich side ( $0.17 \mathrm{M}$ water concentration) (b). Phase plots showing the phase difference between the detector trace and the monitor trace (black lines), while the grey (water) and red (EAN) lines indicate the cmPALS fit to the data. The solid lines in (a) represent the interpolation according to Equation (4).

The repulsive interactions that exist between nanoparticles in common solvents are generally electrostatic or steric in nature [16]. However, these observations suggest that if nanoparticles are successfully stabilized in EAN, electrostatic interparticle interactions may not be expressed because they are assumed to be quenched by the overwhelming ionic environment of the medium. Even though the particle surfaces are charged, conventional models of electrostatic repulsion in water cannot be successfully used in this case, since they are unable to account for such high ionic strengths as in ILs. In such cases, the origin of colloidal stabilization is most likely related to the formation of IL layers at the nanoparticles surface. Nonetheless, electrostatic interactions exist between all the charged species present in the system (anions, cations, and the particle surface charge), and are responsible for the nanoscopic structural organization. As shown in several experimental studies $[6,21,26,33,56]$ and numerical simulations $[57,58]$, such interactions originate from the structuration of IL layers near surfaces.

Furthermore, using the measured electrophoretic mobility values on the water-rich side $C C C_{W}$ values can be calculated based on the DLVO theory [59]. Since the mobility data correlate with the electrokinetic potentials $[17,60]$, the following equation can be used.

$$
\mathrm{CCC}_{W}=\frac{1}{8 \pi l_{B}}\left(\frac{24 \pi}{\operatorname{He\varepsilon \varepsilon }_{0}}\right)^{2 / 3} \sigma^{4 / 3}
$$

where $l_{B}$ is the Bjerrum length, $H$ is the Hamaker constant, $e$ is the base of the natural logarithm, $\varepsilon$ is the dielectric constant, $\varepsilon_{0}$ is the vacuum permittivity, and $\sigma$ is the surface charge density of the particle at the slip plane, which can be determined with the DebyeHückel model [46]. The Hamaker constants used for the calculations was $9 \times 10^{-21} \mathrm{~J}$ [29]. The obtained $C C C_{W}$ values for the SL and SL-IP-2 particles were $0.25 \mathrm{M}$ and $0.14 \mathrm{M}$, respectively, which are in a good agreement with the experimentally determined ones (see Table 1). The results imply that DLVO forces acted between particles in both systems in the water-rich regime (DLVO regime) and that the magnitude of the surface charge determines the $C C C_{W}$, which is higher for SL than for SL-IP-2 (see the mobility magnitudes in Figure 2 at low and high IP-2 doses). 


\subsection{Effect of Added Salt}

Subsequently, the effect of the addition of $\mathrm{NaNO}_{3}$ on the aggregation processes in the particle-EAN systems was studied. It is expected that $\mathrm{Na}^{+}$ion shows stronger influence on the interfacial features, since $\mathrm{NO}_{3}^{-}$ions were already present in the mixtures as a component of EAN (i.e., $\mathrm{NO}_{3}^{-}$was chosen because no additional anions are introduced into the systems in this way). Note that, for the SL particle, $\mathrm{Na}^{+}$was the counterion, while for the SL-IP-2, it acts as coion. Figure 5 shows the obtained aggregation rate trends for both studied latex particle types in the EAN-water mixtures at different concentrations of $\mathrm{NaNO}_{3}$
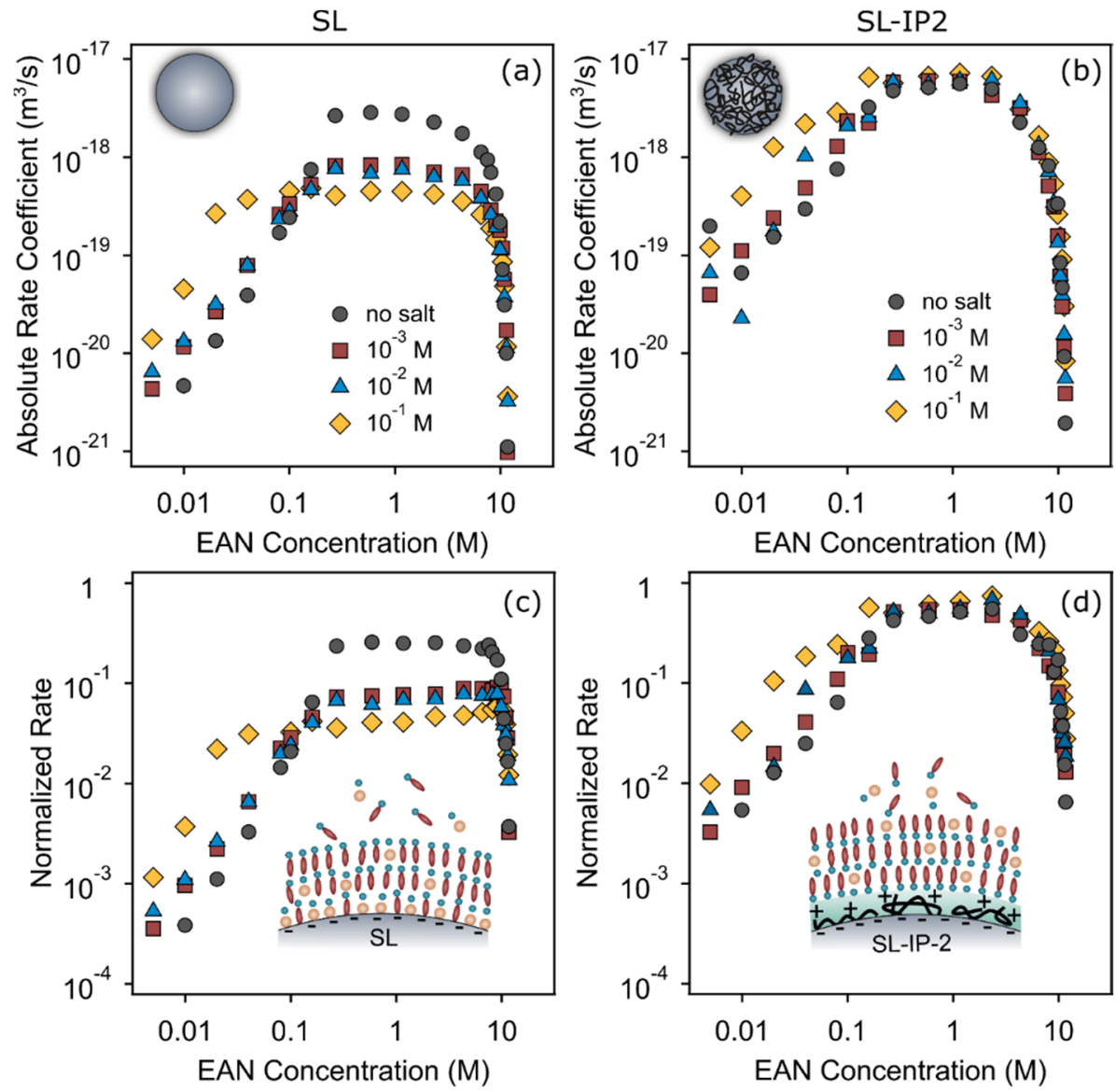

Figure 5. Absolute aggregation rate coefficients $(\mathbf{a}, \mathbf{b})$ and the same quantities normalized to the Smoluchowski rate coefficients (c,d) of SL (left column) and SL-IP-2 particles (right column) versus the EAN concentration in EAN-water mixtures with different concentrations of $\mathrm{NaNO}_{3}$ background electrolyte. The $\mathrm{pH}$ value of the aqueous stock solutions was set to 4 .

The $C C C_{W}$ values tend to decrease for both type of particles with increasing the concentration of $\mathrm{NaNO}_{3}$, in the system (see Table 1). Accordingly, the smallest $\mathrm{CCC}_{W}$ can be observed in the presence of $0.1 \mathrm{M} \mathrm{NaNO}_{3}$, due to the more significant charge screening from the increased amount of counterions, which agrees with the DLVO theory prediction.

Nevertheless, the $C C C_{I L}$ value, which was determined at the IL-rich side, tend to be independent from the presence of $\mathrm{NaNO}_{3}$ for both type of particles, i.e., the obtained values were identical within the experimental error as in previously investigated systems with no salt added. However, the values significantly differ for the two particle types, probably due to the presence of additional steric interactions between the adsorbed IP-2 layers in case of SL-IP-2 particles. Such an additional repulsion led to increased resistance against water induced aggregation. The CCC values deduced from Figure $5 \mathrm{c}, \mathrm{d}$ for SL and SL-IP-2 particles are summarized in Table 1. 
Besides, Figure 5a further indicates that the presence of the $\mathrm{Na}^{+}$ions seems to stabilize the SL dispersions in the intermediate concentration regime in EAN-water system, where the viscous stabilization occurs, since the aggregation rates in the presence of salt ions tend to be almost one order of magnitude smaller than in the case when the simple electrolyte is not present. Another noteworthy observation is that this stabilization is more pronounced with increasing amount of $\mathrm{Na}^{+}$ions, e.g., the aggregation rates were the lowest at the presence of $0.1 \mathrm{M} \mathrm{NaNO}_{3}$. This trend can be partly explained by the accumulation of $\mathrm{Na}^{+}$ions on the negatively charged SL particle surface and thus, they are most probably involved in the development of the initial cationic layer, which compensates the negative charge of the SL. As $\mathrm{Na}^{+}$ions can interact with the EAN components at the interface, they contribute to the formation of ordered solvation layers around the particle and consequently, also to the development of more stable particles. Similar scenario was observed for different solid/IL interfaces in the presence of inorganic ions [33,61]. In general, the influence of added ions can be attributed to their affinity with cations, anions, and the particle surface, to their size compared to that of the components of the IL and to their interaction with water.

Nevertheless, the generic trend in the aggregation rate coefficients in the case of SLIP-2 particles, where the $\mathrm{Na}^{+}$ions have the same sign of charge as the polymer-coated particles, was very similar in all cases, i.e., the above-mentioned $\mathrm{NaNO}_{3}$ concentration dependence on $k$ could not be observed. This fact indicates that the electrostatic repulsion between the co-ions and the charged particle may lead to very weak adsorption, therefore $\mathrm{Na}^{+}$ions interact only slightly with the particles of the same sign of charge and hence, their influence on the particle surface charge is negligible. As a result, the aggregation features of the SL-IP-2 particles are basically independent of the presence of the background salt and the obtained fast aggregation rates remain unchanged within the experimental uncertainty regardless of the $\mathrm{NaNO}_{3}$ concentration.

\section{Conclusions}

Results of time-resolved light scattering measurements performed in aqueous EAN solutions revealed that the aggregation of bare and polymer functionalized latex particles is governed by electrostatic forces in the diluted IL samples, where dissociated and hydrated IL ions are present. The particle aggregation slows down at higher IL concentrations due to the increased viscosity of the medium. Besides, the particles were highly stable in pure ILs, where only a small amount of water was present. Under the latter experimental conditions, no significant change in the particle movement could be detected with applying an electric field in electrophoretic experiments. This indicates that the prevailing nature of colloidal stability of these systems is not electrostatic as in diluted IL solutions and that the dispersions are stable, rather due to repulsive oscillatory forces resulting from the layered assembly of the IL constituents on the particle surface. These results shed light on the origin of the main interparticle forces responsible for the colloidal stability of the particles in both pure ILs and in their aqueous solutions.

Furthermore, the effect of added $\mathrm{NaNO}_{3}$ to the system was also studied. The results indicate that the $\mathrm{Na}^{+}$ions change the interfacial assembly of IL ions in the studied system and influence the stability of the colloidal dispersions. Such influence may be more or less significant, depending on the nature of the added ions (size, affinity to the surface and the solvation level of the ions) and the surface charge of the particles. In conclusion, the organization of the IL constituents at the particle surface as well as the interparticle forces can be altered by adding the appropriate type and amount of salt, which is particularly important once particle-IL dispersions are designed for certain applications. Knowing the physico-chemical parameters of the particles and ILs applied, the stability regimes can be qualitatively predicted based on the present findings. 


\begin{abstract}
Author Contributions: Conceptualization, I.S. and M.T.; methodology, D.T., I.S. and M.T.; software, I.S. and M.T.; validation, D.T., I.S. and M.T.; formal analysis, D.T., I.S. and M.T.; investigation, D.T., I.S. and M.T.; data curation, D.T., I.S. and M.T.; writing—original draft preparation, D.T.; writing—review and editing, I.S. and M.T.; visualization, D.T., I.S. and M.T.; supervision, I.S.; project administration, I.S. and M.T.; funding acquisition, I.S. and M.T. All authors have read and agreed to the published version of the manuscript.
\end{abstract}

Funding: The project was financed by the Hungarian National Research, Development and Innovation Office (SNN131558) and the Slovenian Research Agency (research core funding No. P1-0201 and project No. N1-0139 “Delamination of Layered Materials and Structure-Dynamics Relationship in Green Solvents"). D. T. was supported by the ÚNKP-21-3-SZTE-413 New National Excellence Program of the Ministry for Innovation and Technology from the source of the National Research, Development and Innovation Fund.

Institutional Review Board Statement: Not relevant.

Informed Consent Statement: Not relevant.

Data Availability Statement: Not relevant.

Conflicts of Interest: The authors declare no conflict of interest.

\title{
References
}

1. Armand, M.; Endres, F.; MacFarlane, D.R.; Ohno, H.; Scrosati, B. Ionic-liquid materials for the electrochemical challenges of the future. Nat. Mater. 2009, 8, 621-629. [CrossRef] [PubMed]

2. Donato, K.Z.; Matejka, L.; Mauler, R.S.; Donato, R.K. Recent applications of ionic liquids in the sol-gel process for polymer-silica nanocomposites with ionic interfaces. Colloids Interfaces 2017, 1, 5. [CrossRef]

3. Franca, J.M.P.; Vieira, S.I.C.; Lourenco, M.J.V.; Murshed, S.M.S.; de Castro, C.A.N. Thermal conductivity of $\mathrm{C}_{4} \mathrm{mim}\left(\mathrm{CF}_{3} \mathrm{SO}_{2}\right)_{2} \mathrm{~N}$ and $\mathrm{C}_{2}$ mim $\mathrm{EtSO}_{4}$ and their ionanofluids with carbon nanotubes: Experiment and theory. J. Chem. Eng. Data 2013, 58, 467-476. [CrossRef]

4. Minea, M.A.; Murshed, S.M.S. Ionic liquids-based nanocolloids-A review of progress and prospects in convective heat transfer applications. Nanomaterials 2021, 11, 1039. [CrossRef] [PubMed]

5. He, Z.Q.; Alexandridis, P. Ionic liquid and nanoparticle hybrid systems: Emerging applications. Adv. Colloid Interface Sci. 2017, 244, 54-70. [CrossRef]

6. Ueno, K.; Watanabe, M. From colloidal stability in ionic liquids to advanced soft materials using unique media. Langmuir 2011, 27, 9105-9115. [CrossRef]

7. Tunckol, M.; Durand, J.; Serp, P. Carbon nanomaterial-ionic liquid hybrids. Carbon 2012, 50, 4303-4334. [CrossRef]

8. Parvulescu, V.I.; Hardacre, C. Catalysis in ionic liquids. Chem. Rev. 2007, 107, 2615-2665. [CrossRef]

9. Hallett, J.P.; Welton, T. Room-temperature ionic liquids: Solvents for synthesis and catalysis. 2. Chem. Rev. 2011, 111, 3508-3576. [CrossRef]

10. Taskin, M.; Cognigni, A.; Zirbs, R.; Reimhult, E.; Bica, K. Surface-active ionic liquids for palladium-catalysed cross coupling in water: Effect of ionic liquid concentration on the catalytically active species. RSC Adv. 2017, 7, 41144-41151. [CrossRef]

11. Le Bideau, J.; Viau, L.; Vioux, A. Ionogels, ionic liquid based hybrid materials. Chem. Soc. Rev. 2011, 40, 907-925. [CrossRef] [PubMed]

12. Silvester, D.S.; Jamil, R.; Doblinger, S.; Zhang, Y.X.; Atkin, R.; Li, H. Electrical double layer structure in ionic liquids and its importance for supercapacitor, battery, sensing, and lubrication applications. J. Phys. Chem. C 2021, 125, 13707-13720. [CrossRef]

13. Beattie, D.A.; Espinosa-Marzal, R.M.; Ho, T.T.M.; Popescu, M.N.; Ralston, J.; Richard, C.J.E.; Sellapperumage, P.M.F.; Krasowska, M. Molecularly-thin precursor films of imidazolium-based ionic liquids on mica. J. Phys. Chem. C 2013, 117, 23676-23684. [CrossRef]

14. Mezger, M.; Schroder, H.; Reichert, H.; Schramm, S.; Okasinski, J.S.; Schoder, S.; Honkimaki, V.; Deutsch, M.; Ocko, B.M.; Ralston, J.; et al. Molecular layering of fluorinated ionic liquids at a charged sapphire (0001) surface. Science 2008, 322, $424-428$. [CrossRef] [PubMed]

15. Elimelech, M.; Gregory, J.; Jia, X.; Williams, R.A. Particle Deposition and Aggregation: Measurement, Modeling, and Simulation; Butterworth-Heinemann Ltd.: Oxford, UK, 1995.

16. Israelachvili, J. Intermolecular and Surface Forces, 3rd ed.; Academic Press: London, UK, 2011.

17. Russel, W.B.; Saville, D.A.; Schowalter, W.R. Colloidal Dispersions; Cambridge University Press: Cambridge, UK, 1989.

18. Ueno, K.; Inaba, A.; Kondoh, M.; Watanabe, M. Colloidal stability of bare and polymer-grafted silica nanoparticles in ionic liquids. Langmuir 2008, 24, 5253-5259. [CrossRef] [PubMed]

19. Asencio, R.A.; Cranston, E.D.; Atkin, R.; Rutland, M.W. Ionic liquid nanotribology: Stiction suppression and surface induced shear thinning. Langmuir 2012, 28, 9967-9976. [CrossRef] 
20. Hjalmarsson, N.; Atkin, R.; Rutland, M.W. Is the boundary layer of an ionic liquid equally lubricating at higher temperature? Phys. Chem. Chem. Phys. 2016, 18, 9232-9239. [CrossRef]

21. Smith, J.A.; Werzer, O.; Webber, G.B.; Warr, G.G.; Atkin, R. Surprising particle stability and rapid sedimentation rates in an ionic liquid. J. Phys. Chem. Lett. 2010, 1, 64-68. [CrossRef]

22. Szilagyi, I.; Szabo, T.; Desert, A.; Trefalt, G.; Oncsik, T.; Borkovec, M. Particle aggregation mechanisms in ionic liquids. Phys. Chem. Chem. Phys. 2014, 16, 9515-9524. [CrossRef] [PubMed]

23. Hayes, R.; Warr, G.G.; Atkin, R. Structure and nanostructure in ionic liquids. Chem. Rev. 2015, 115, 6357-6426. [CrossRef]

24. Hayes, R.; Imberti, S.; Warr, G.G.; Atkin, R. The nature of hydrogen bonding in protic ionic liquids. Angew. Chem. Int. Ed. 2013, 52, 4623-4627. [CrossRef]

25. Hao, J.C.; Zemb, T. Self-assembled structures and chemical reactions in room-temperature ionic liquids. Curr. Opin. Colloid Interface Sci. 2007, 12, 129-137. [CrossRef]

26. Werzer, O.; Cranston, E.D.; Warr, G.G.; Atkin, R.; Rutland, M.W. Ionic liquid nanotribology: Mica-silica interactions in ethylammonium nitrate. Phys. Chem. Chem. Phys. 2012, 14, 5147-5152. [CrossRef] [PubMed]

27. Elbourne, A.; Voitchovsky, K.; Warr, G.G.; Atkin, R. Ion structure controls ionic liquid near-surface and interfacial nanostructure. Chem. Sci. 2015, 6, 527-536. [CrossRef]

28. Sheehan, A.; Jurado, L.A.; Ramakrishna, S.N.; Arcifa, A.; Rossi, A.; Spencer, N.D.; Espinosa-Marzal, R.M. Layering of ionic liquids on rough surfaces. Nanoscale 2016, 8, 4094-4106. [CrossRef] [PubMed]

29. Oncsik, T.; Desert, A.; Trefalt, G.; Borkovec, M.; Szilagyi, I. Charging and aggregation of latex particles in aqueous solutions of ionic liquids: Towards an extended Hofmeister series. Phys. Chem. Chem. Phys. 2016, 18, 7511-7520. [CrossRef] [PubMed]

30. Katana, B.; Takács, D.; Csapo, E.; Szabo, T.; Jamnik, A.; Szilagyi, I. Ion specific effects on the stability of halloysite nanotube colloids-Inorganic salts versus ionic liquids. J. Phys. Chem. B 2020, 124, 9757-9765. [CrossRef]

31. Katana, B.; Takács, D.; Szerlauth, A.; Sáringer, S.; Varga, G.; Jamnik, A.; Bobbink, F.D.; Dyson, P.J.; Szilagyi, I. Aggregation of halloysite nanotubes in the presence of multivalent ions and ionic liquids. Langmuir 2021, 37, 11869-11879. [CrossRef] [PubMed]

32. Riedl, J.C.; Kazemi, M.A.A.; Cousin, F.; Dubois, E.; Fantini, S.; Lois, S.; Perzynski, R.; Peyre, V. Colloidal dispersions of oxide nanoparticles in ionic liquids: Elucidating the key parameters. Nanoscale Adv. 2020, 2, 1560-1572. [CrossRef]

33. Nordstrom, J.; Aguilera, L.; Matic, A. Effect of lithium salt on the stability of dispersions of fumed silica in the ionic liquid $\mathrm{BMImBF}_{4}$. Langmuir 2012, 28, 4080-4085. [CrossRef] [PubMed]

34. Han, M.W.; Espinosa-Marzal, R.M. Influence of water on structure, dynamics, and electrostatics of hydrophilic and hydrophobic ionic liquids in charged and hydrophilic confinement between mica surfaces. ACS Appl. Mater. Interfaces 2019, 11, 33465-33477. [CrossRef]

35. Hayes, R.; Borisenko, N.; Corr, B.; Webber, G.B.; Endres, F.; Atkin, R. Effect of dissolved LiCl on the ionic liquid-Au(111) electrical double layer structure. Chem. Commun. 2012, 48, 10246-10248. [CrossRef]

36. Katana, B.; Takács, D.; Bobbink, F.D.; Dyson, P.J.; Alsharif, N.B.; Tomšič, M.; Szilagyi, I. Masking specific effects of ionic liquid constituents at the solid-liquid interface by surface functionalization. Phys. Chem. Chem. Phys. 2020, 22, 24764-24770. [CrossRef]

37. Takács, D.; Katana, B.; Szerlauth, A.; Sebők, D.; Tomšič, M.; Szilagyi, I. Influence of adsorption of ionic liquid constituents on the stability of layered double hydroxide colloids. Soft Matter 2021, 17, 9116-9124. [CrossRef] [PubMed]

38. Zhong, W.; Bobbink, F.D.; Fei, Z.F.; Dyson, P.J. Polyimidazolium salts: Robust catalysts for the cycloaddition of carbon dioxide into carbonates in solvent-free conditions. ChemSusChem 2017, 10, 2728-2735. [CrossRef] [PubMed]

39. Zarrougui, R.; Dhahbi, M.; Lemordant, D. Transport and thermodynamic properties of ethylammonium nitrate-water binary mixtures: Effect of temperature and composition. J. Solut. Chem. 2015, 44, 686-702. [CrossRef]

40. Holthoff, H.; Egelhaaf, S.U.; Borkovec, M.; Schurtenberger, P.; Sticher, H. Coagulation rate measurements of colloidal particles by simultaneous static and dynamic light scattering. Langmuir 1996, 12, 5541-5549. [CrossRef]

41. Berne, B.J.; Pecora, R. Dynamic Light Scattering; Robert E. Krieger Publishing: Malabar, FL, USA, 1990.

42. Cao, T.C.; Borkovec, M.; Trefalt, G. Heteroaggregation and homoaggregation of latex particles in the presence of alkyl sulfate surfactants. Colloids Interfaces 2020, 4, 52. [CrossRef]

43. Yu, W.L.; Matijevic, E.; Borkovec, M. Absolute heteroaggregation rate constants by multiangle static and dynamic light scattering. Langmuir 2002, 18, 7853-7860. [CrossRef]

44. Hierrezuelo, J.; Vaccaro, A.; Borkovec, M. Stability of negatively charged latex particles in the presence of a strong cationic polyelectrolyte at elevated ionic strengths. J. Colloid Interface Sci. 2010, 347, 202-208. [CrossRef]

45. Rouster, P.; Dondelinger, M.; Galleni, M.; Nysten, B.; Jonas, A.M.; Glinel, K. Layer-by-layer assembly of enzyme-loaded halloysite nanotubes for the fabrication of highly active coatings. Colloid Surf. B 2019, 178, 508-514. [CrossRef]

46. Trefalt, G.; Szilagyi, I.; Borkovec, M. Poisson-Boltzmann description of interaction forces and aggregation rates involving charged colloidal particles in asymmetric electrolytes. J. Colloid Interface Sci. 2013, 406, 111-120. [CrossRef] [PubMed]

47. Oncsik, T.; Trefalt, G.; Borkovec, M.; Szilagyi, I. Specific ion effects on particle aggregation induced by monovalent salts within the Hofmeister series. Langmuir 2015, 31, 3799-3807. [CrossRef] [PubMed]

48. Oncsik, T.; Trefalt, G.; Csendes, Z.; Szilagyi, I.; Borkovec, M. Aggregation of negatively charged colloidal particles in the presence of multivalent cations. Langmuir 2014, 30, 733-741. [CrossRef] [PubMed]

49. Valmacco, V.; Trefalt, G.; Maroni, P.; Borkovec, M. Direct force measurements between silica particles in aqueous solutions of ionic liquids containing 1-butyl-3-methylimidazolium (BMIM). Phys. Chem. Chem. Phys. 2015, 17, 16553-16559. [CrossRef] 
50. Sinha, P.; Szilagyi, I.; Ruiz-Cabello, F.J.M.; Maroni, P.; Borkovec, M. Attractive forces between charged colloidal particles induced by multivalent ions revealed by confronting aggregation and direct force measurements. J. Phys. Chem. Lett. 2013, 4, 648-652. [CrossRef]

51. Kobayashi, M.; Juillerat, F.; Galletto, P.; Bowen, P.; Borkovec, M. Aggregation and charging of colloidal silica particles: Effect of particle size. Langmuir 2005, 21, 5761-5769. [CrossRef]

52. Vanecht, E.; Binnemans, K.; Patskovsky, S.; Meunier, M.; Seo, J.W.; Stappers, L.; Fransaer, J. Stability of sputter-deposited gold nanoparticles in imidazolium ionic liquids. Phys. Chem. Chem. Phys. 2012, 14, 5662-5671. [CrossRef]

53. Rubim, J.C.; Trindade, F.A.; Gelesky, M.A.; Aroca, R.F.; Dupont, J. Surface-enhanced vibrational spectroscopy of tetrafluoroborate 1-n-butyl-3-methylimidazolium $\left(\mathrm{BMIBF}_{4}\right)$ ionic liquid on silver surfaces. J. Phys. Chem. C 2008, 112, 19670-19675. [CrossRef]

54. Guibert, C.; Dupuis, V.; Fresnais, J.; Peyre, V. Controlling nanoparticles dispersion in ionic liquids by tuning the pH. J. Colloid Interface Sci. 2015, 454, 105-111. [CrossRef]

55. Lopez-Leon, T.; Jodar-Reyes, A.B.; Bastos-Gonzalez, D.; Ortega-Vinuesa, J.L. Hofmeister effects in the stability and electrophoretic mobility of polystyrene latex particles. J. Phys. Chem. B 2003, 107, 5696-5708. [CrossRef]

56. Hayes, R.; Warr, G.G.; Atkin, R. At the interface: Solvation and designing ionic liquids. Phys. Chem. Chem. Phys. 2010, 12, 1709-1723. [CrossRef]

57. Canova, F.F.; Matsubara, H.; Mizukami, M.; Kurihara, K.; Shluger, A.L. Shear dynamics of nanoconfined ionic liquids. Phys. Chem. Chem. Phys. 2014, 16, 8247-8256. [CrossRef]

58. Feng, G.A.; Qiao, R.; Huang, J.S.; Dai, S.; Sumpter, B.G.; Meunier, V. The importance of ion size and electrode curvature on electrical double layers in ionic liquids. Phys. Chem. Chem. Phys. 2011, 13, 1152-1161. [CrossRef] [PubMed]

59. Galli, M.; Saringer, S.; Szilagyi, I.; Trefalt, G. A simple method to determine critical coagulation concentration from electrophoretic mobility. Colloids Interfaces 2020, 4, 20. [CrossRef]

60. Delgado, A.V.; Gonzalez-Caballero, F.; Hunter, R.J.; Koopal, L.K.; Lyklema, J. Measurement and interpretation of electrokinetic phenomena. J. Colloid Interface Sci. 2007, 309, 194-224. [CrossRef] [PubMed]

61. Mamusa, M.; Siriex-Plenet, J.; Cousin, F.; Dubois, E.; Peyrea, V. Tuning the colloidal stability in ionic liquids by controlling the nanoparticles/liquid interface. Soft Matter 2014, 10, 1097-1101. [CrossRef] [PubMed] 